\title{
How Do Students React When Their Performance Is Worse or Better Than Expected?
}

\author{
An Analysis Based on the ViolEx Model
}

\author{
Martin PinquartiD, Julia C. Koß, and Helena Block \\ Department of Psychology, Philipps-University of Marburg
}

\begin{abstract}
We analyzed grade level and sex differences for changes in expectations and coping with expectation violations, based on the ViolEx model. A sample of 114 third to sixth graders (male, $n=44,38.6 \%$; female, $n=70,61.4 \%$ ) reported their expected achievement in trials with no feedback, worse-than-expected achievement feedback, and better-than-expected feedback. Expectations improved across the no-feedback and better-than-expected feedback trials, and deteriorated across worse-than-expected trials. While expectation change did not vary by grade level or sex, reported coping with expectation violation did vary, and pupils of younger years reported higher attempts to fulfill their expectations (assimilative behavior). Immunization against worse-than-expected feedback was associated with higher expectations in the negative-feedback condition, but protective effects of immunization were lost with an increasing number of expectation violations. In addition, higher willingness to reduce one's expectations (accommodation) inhibited the update of expectations after receiving better-than-expected feedback. Conclusions for future research are drawn.
\end{abstract}

Keywords: expectation violation, immunization, assimilation, accommodation, ViolEx model

Wie reagieren Schüler, wenn ihre Leistung schlechter oder besser als erwartet ausfällt? Eine Analyse auf Basis des ViolEx-Modells

Zusammenfassung: Untersucht wurden Klassenstufen- und Geschlechtsunterschiede in Erwartungsveränderungen und dem Umgang mit Erwartungsverletzungen basierend auf dem ViolEx Modell. Dritt- bis Sechstklässler $(N=114)$ gaben Leistungserwartungen in Versuchsdurchgängen ohne Leistungsrückmeldung sowie nach schlechter und besser als erwartetem Feedback an. Die Erwartungen verbesserten sich in Durchgängen ohne Feedback und mit positivem Feedback und verschlechterten sich nach negativem Feedback. Während Erwartungsänderungen nicht mit Geschlecht und Klassenstufe variierten, berichteten jüngere Schüler mehr Versuche, ihr Erwartung zu erfüllen (Assimilation). Immunisierung gegen unerwartetes negatives Feedback ging mit geringeren Erwartungsänderungen einher, aber dieser Effekt verschwand nach wiederholt negativem Feedback. Eine größere berichtete Bereitschaft zur Anpassung von Erwartungen (Akkommodation) sagte das entsprechende Verhalten vorher. Abschließend werden Schlussfolgerungen für die weitere Forschung gezogen.

Schlüsselwörter: Erwartungsverletzung, Immunisierung, Assimilation, Akkommodation, ViolEx Modell

Children and adolescents build expectations about their future achievement (e.g., Hacker et al., 2000; Pinquart \& Ebeling, 2020). Even if expectations are to some extent based on previous achievement, young people often experience that their achievement is better or worse than expected (with overly optimistic expectations being common in the context of school and sports; Pinquart \& Ebeling, 2020; Scanlan \& Passer, 1981). Although disconfirmed expectations may lead to change of expectations about future achievement, overly optimistic achievement expectations often persist over time despite experiencing expectation violations (Kruger \& Dunning, 1999; Pinquart \& Ebeling, 2020). The ViolEx (violated expectation) model has been developed for explaining persistence and change of expectations after being confronted with expectationdisconfirming information (Gollwitzer et al., 2018; Rief \& Glombiewski, 2016). It proposes that individuals react to expectation violations in three different ways. First, they may immunize themselves against the unexpected event by devaluing the discrepant information, such as ignoring it or doubting its validity or relevance (e.g., perceiving it as an exception to the rule). Second, they may take an active role in bringing the reality in concordance with their expectation and reduce the risk for future expectation disconfirmation (assimilation). For example, after receiving a worse-than-expected mark on an achievement 
test, students may try to work harder for a better (expected) grade on the next test. Third, they may change their expectation so that it matches the (previously unexpected) outcome and will be in accordance with such outcomes in future situations (accommodation). For example, students may lower their expectation for the next test so that their expectation will come true more easily. Some of these processes have also been included in other models of coping with expectation violation, under different labels (e.g., ignoring, subtyping, revising expectations; Roese \& Sherman, 2007). Although using a particular way of coping is probably often a spontaneous, automatic, and uncontrollable process, individuals can later report on their use and its effects. The ViolEx model proposes that immunization and assimilation promote the persistence of expectations, while accommodation leads to expectation change (Gollwitzer et al., 2018).

When focusing on children and adolescents, it is of interest whether achievement-related expectations and the proposed three processes of coping with expectation violation differ by age, sex, and other characteristics of the child, and which of these coping processes explain persistence and change of the expectation after being confronted with expectation violations. The present study analyzed grade level and sex differences in change of achievement expectations in an attention task in which students received no achievement feedback first, followed by worse-than-expected, and better-than-expected achievement feedback.

\section{Age and Sex Differences in Reactions to Disconfirmed Achievement Expectations}

There is some empirical evidence that overly optimistic achievement expectations are particularly common in preschoolers and in the first years of elementary school, while older students are more likely to adjust their expectations in response to their achievements (Muenks et al., 2018). Younger children's lower adjustments of expectations after receiving negative feedback may be due to their limited cognitive abilities to differentiate between hopes or aspirations and the chance of succeeding (Muenks et al., 2018). For example, Parsons and Ruble (1977) found that, after receiving negative performancerelated feedback, 3- to 5-year-olds had more positive expectations than 6- to 8-year-olds and 9- to 11-year-olds, while the expectations of the latter two groups did not differ. Stipek and Hoffman (1980a) observed that 5- to 6year-old girls had higher expectations than 7- to 8-year- olds after receiving negative feedback. A recent metaanalysis indicates that students' expectations exceed prior achievement less frequently if the students are older (Pinquart \& Ebeling, 2020).

However, results of available studies are, in part, inconsistent. For example, Stipek and Hoffman (1980b) showed that compared with high-achieving peers, lowachieving third graders adjusted their expectations for future achievement less often than low-achieving first graders did. In a study of first to fourth graders, Pinquart and Block (2020) found that immediately after the confrontation with a worse-than-expected achievement, younger students changed their expectations more than older students did. After giving some time to think about possible reasons for their unexpected achievement, younger students increased their expectations for their achievement in the next round, while older students showed a further drop in achievement expectation.

With regard to sex differences, Stipek and Hoffman (1980a) reported that after receiving negative feedback about their achievement, 7- to 8-year-old boys had higher success expectations than girls of the same age. No sex differences in expectations were found after receiving positive achievement-related feedback. Parsons and Ruble (1977) found that 6- to 8-year-old girls were more likely to adjust their expectations than boys, after receiving negative as well as positive achievement feedback. The authors suggested that sex differences in socialization may make girls more sensitive to negative achievement-related feedback. However, a recent study found no sex differences for expectation change after a worse-thanexpected achievement (Pinquart \& Block, 2020). Thus, boys' and girls' expectation changes in response to achievement-related feedback might have become more similar in recent years.

If younger children and boys update their expectations less than older children and girls, they should, according to the ViolEx model, report lower levels of accommodation and higher levels of immunization against expectation-disconfirming feedback and/or assimilative behavior aimed at confirming their expectation (Gollwitzer et al., 2018). Until now, only one study assessed these coping processes among children. With the method of thinking aloud (i.e., verbalizing one's thoughts aloud), Pinquart and Block (2020) coded verbal responses after a worsethan-expected achievement with regard to whether they reflected immunization, assimilation, or accommodation. The responses did not vary by age or sex, but it should be noted that very few reports of assimilation and accommodation were identified, which reduced the chance of finding significant differences. 


\section{Associations of the ViolEx Processes With Expectation Change}

Most available research on effects of immunization, assimilation, and accommodation (and related concepts) on expectation change has been conducted with adults. With regard to the processes suggested to contribute to the persistence of expectations, this research focused on immunization rather than assimilation (e.g., Filipowicz et al., 2018; Kube et al., 2018 </litr>; Roese \& Sherman, 2007).

In the study by Pinquart and Block (2020), children's reported immunization against the negative discrepant feedback was followed by less frequent expectation change. In contrast, reported assimilative and accommodative tendencies did not predict expectation change. Immunization may have been easy to apply in that study because the children were only confronted with a lowerthan-expected achievement in one trial, and due to the study design, participants could not increase the chance of achievement expectations being fulfilled by using assimilative behavior.

\section{Research Questions}

In the present study, students first completed trials of an attention task with no achievement feedback, followed by trials with feedback indicating worse-than-expected achievement, and trials with better-than-expected achievement. The dependent variable was the time expected for completing the next trial. Completing a number of similar trials leads to an increase in performance over time because the performances in the first trial result in an activation boost that makes the repeated task performance more efficient (Sohn \& Anderson, 2001). This repetition effect may lead to a decrease in the expected time for task completion even if no explicit achievement feedback is given. The direction of expectation change in the feedback conditions was expected to depend upon whether achievement was better or worse than expected, although individuals might show stronger expectation change if the achievement was better than expected compared with worse than expected (optimistic update bias; Garrett \& Sharot, 2017). Thus, Hypothesis 1 stated that the expected time for completing the task would decline in the no-feedback condition and in the betterthan-expected condition, while the expected time would increase after getting the feedback that one needed more time than expected in the previous trial.

Due to that fact that after expectation violations, some studies have found less frequent expectation change in younger compared with older children, and in boys compared with girls (Parsons \& Ruble, 1977; Stipek \& Hoffman, 1980a), Hypothesis 2 asked whether the present study would replicate these age and/or grade level and sex differences. Because the ViolEx model assumes that immunization and assimilation inhibit expectation change while accommodation indicates expectation change (Gollwitzer et al., 2018; Rief \& Glombiewski, 2016), the hypothesized age and/or grade level and sex differences in expectation change would indicate that there were also related differences in the processes of coping with expectation violation. Thus, Hypothesis 3 stated that children from younger grade levels and boys would report higher levels of immunization and assimilation, and lower levels of accommodation than older children and girls.

In the only study that has analyzed the effects of children's three ViolEx strategies on expectation change, Pinquart and Block (2020) found only an effect of immunization. However, the ViolEx model assumes that assimilation and (reported) accommodation should also relate to the persistence and change of expectations (Gollwitzer et al., 2018; Rief \& Glombiewski, 2016). Immunization and assimilation would be especially relevant for protecting the expectation if the achievement was worse than expected (Garrett \& Sharot, 2017). To test this assumption, Hypothesis 4 stated that, after worse-thanexpected achievement, higher reported levels of immunization and assimilation would predict lower expectation change, while higher reported levels of accommodation would relate to higher expectation change. We did not state a hypothesis about the role of the ViolEx processes after the better-than-expected achievement, because under this condition, students are likely to be willing to update (improve) their expectation rather than to stabilize it (Garrett \& Sharot, 2017). Finally, if grade level and sex differences in expectation change and in the use of immunization, assimilation, and accommodation were identified (according to Hypotheses 2 and 3), Hypothesis 5 postulated that the grade level and sex differences in expectation change would be mediated by related differences in immunization, assimilation, and accommodation.

\section{Methods}

\section{Participants}

An experimental study was conducted with third to sixth graders from a German town and its surroundings. We did not include students from grades 1 and 2, because pretests 
showed that they had difficulties with providing consistent reports on the ViolEx strategies, using rating scales. After the consent of a parent or guardian was obtained, the data were collected at local schools in individual test sessions, which lasted about 30 minutes per child. The ethical review board of the research institute of the authors (No. 2019-01k) approved the study. Because in our previous research, larger numbers of parents did not want their child to participate if the anonymized data was to be made available to third persons, we decided not to ask them for their informed consent to make the data available to the scientific community.

In total, 122 students were recruited. Due to an initial software error of the computer game, eight students had to be excluded from the statistical analysis, as the provision of achievement feedback did not work properly. Of the remaining 114 participants, 44 (38.6\%) were male, and $70(61.4 \%)$ were female students. They had a mean age of 10.07 years $(S D=1.31)$. In total, 30 third graders, 28 fourth graders, 28 fifth graders, and 28 sixth graders participated in this study.

\section{Procedure}

\section{Expectation Violation Paradigm}

As part of a computer game, nine fields were presented on the screen where different shapes (crosses and circles) appeared in different numbers and combinations (see Electronic Supplementary Material, ESM 1). The participants were asked to press the space bar whenever a predefined combination of the shapes (target) was shown. The aim of the game was to get 10 hits as quickly as possible and make as few mistakes (omissions or false positives) as possible. At the start, participants received an anchor range of the average expected time of other students, for orientation. Before each trial, we asked the participants for their expected time to get 10 hits (in seconds). In the first four trials (the no-feedback condition), the students did not get feedback about their time needed for completing the task. In the next four trials (the negative-feedback condition), the number of targets that occurred was manipulated by the computer so that the actual time needed for 10 correct hits was about 10-20\% above the child's expectations. At the end of each trial, the time for completing the trial was shown on the computer screen immediately after each trial. In the third block (the positive-feedback condition), the number of targets was manipulated so that the students needed about 10-20\% less time than expected, and they got the feedback of being faster than expected. To save time, the positivefeedback condition consisted of only two trials. At the end, students received their favorite out of five rewards (e.g., games, books, toys).

\section{Coping With Expectation Violation}

The questions about coping with expectation violations were asked after the fourth trial of the negative-feedback condition. As no standardized measure for assessing the use of immunization, assimilation, and accommodation after expectation violation was available for children, we developed our own self-report instrument for the present study. The measure was based on the definitions of the three coping strategies by Gollwitzer et al. (2018) and Rief and Glombiewski (2016), and on reports from a previous study of elementary school students on open questions about their reactions after performing worse than expected (Pinquart \& Block, 2020). We formulated eight items for each coping style (see ESM 2). Sample items were "It was an accident that I was slow in the last rounds of the game" (immunization), "Next time I will try harder" (assimilation), and "It's ok for me to need more time in the next rounds" (accommodation). We used the following instructions: "I will now read to you answers from children who were disappointed by some results of the game. Please decide whether you, if you were disappointed by a result, thought that way or not." The items were answered on a five-point Likert scale ranging from not true (coded as 0 ) to very true (coded as 4 ). For easier use, descriptions of the response options were combined with smileys. In the process of formulating items, we had also thought about stating them with no reference to the direction of expectation violation, thus including coping with worse-than-expected and better-than-expected events. However, we had difficulty finding enough of such items, and neutrally formulated items may be less predictive than those addressing coping with worse-thanexpected or better-than-expected events because the reactions to both kinds of events may differ (Garrett \& Sharot, 2017; Moser \& Schroder, 2012).

We conducted an exploratory factor analysis. Visual inspection of the scree plot supported the suggested threefactor solution. Two items on immunization, one on accommodation, and two on assimilation had to be excluded due to factor loadings $<.40$. The scales based on the remaining items (ESM 3) had acceptable internal consistencies (immunization: Cronbach's $\alpha=.76$, assimilation: $\alpha=.78$, accommodation: $\alpha=.75$; ESM 2 and ESM 3). For validation of the coping scales, we collected parental reports on students' flexible goal adjustment and tenacious goal pursuit (based on Lessing et al., 2015) that have been shown to correlate with accommodative coping in elementary school (Lessing et al., 2015). Students' self-reports on assimilative coping with expectation violation correlated with parental reports on tenacious 
Table 1. Expectation change over time (level 1 model)

\begin{tabular}{lccccccccc}
\hline & \multicolumn{3}{c}{ No-feedback condition } & \multicolumn{3}{c}{ Negative-feedback condition } & \multicolumn{3}{c}{ Positive-feedback condition } \\
& Estimate & $t$ & $p$ & Estimate & $t$ & $p$ & Estimate & $t$ & $p$ \\
\hline Time of measurement & -0.97 & -4.63 & .001 & 5.39 & 15.10 & .001 & -5.50 & -8.08 & .001 \\
Constant & 23.80 & 30.17 & .001 & 16.29 & 17.90 & .001 & 46.29 & 32.90 & .001 \\
\hline
\end{tabular}

Note. $t=$ test for statistical significance. In all analyses, the number of degrees of freedom was 114 .

goal pursuit of their child $(r=.23, p<.02, N=106)$. Parental reports on tenacious goal pursuit also showed a negative correlation with student self-reports on accommodation $(r=-.21, p<.04, N=106)$, while parental reports on students' flexible goal adjustment were not significantly correlated with student self-reports on accommodation $(r=.08, p<.42$; ESM 4$)$.

\section{Results}

Power analysis indicated that between 20 and 100 participants were needed for finding the main effects of negative feedback and sex as well as an interaction effect of grade level and time of measurement with a statistical power of .8 and $\alpha=.05$, which have been reported in previous studies (Pinquart \& Block, 2020; Parsons \& Ruble, 1977; Stipek \& Hoffman, 1980a). However, a sample size of 120 would be needed for replicating the effect size of immunization from Pinquart and Block (2020).

All analyses were performed using IBM SPSS (v26.0). Means, standard deviations, and correlations of the study variables are shown in ESM 4 and 5. Mixed linear modeling (MLM) with maximum likelihood estimation was used to analyze changes in expectations over time (Singer \& Willett, 2003). There are two levels of MLM models. The level 1 model (the unconditional growth model) estimates the average within-person initial status and rate of change over time, with time of measurement being the independent variable. The level 2 model captures whether the rate of change and the withinsubjects intercepts of level 1 are predicted by betweensubjects variables at level 2, namely grade level and sex, or the styles of coping with expectation violation, respectively.

One of the advantages of MLM is the ability to specify the within-individual error covariance structure that fits the data best, as choosing an appropriate covariance structure for the growth curve model reduces error variance. The unstructured covariance structure model is most commonly found in longitudinal data and often provides the best fit as it is the most parsimonious, which requires no assumption about the error structure (Singer, 1998). For the models described above, three multiple repeated (level 1) covariance structures were tested (unstructured; compound symmetric - assuming homogeneous variances and constant correlations across all times of measurement; and autoregressive - assuming homogeneous variances and correlations that decline exponentially with temporal distance). The unstructured covariance model was found to show the smallest values in the three fit criteria (-2 log-likelihood, Akaike information criterion, Bayesian information criterion), thus indicating the best fit.

Because we expected different directions of expectation change in the no-feedback, negative feedback, and positive feedback conditions, we computed separate MLM analyses on each condition. Table 1 shows the results from the level 1 model. In the no-feedback condition, the significant slope indicates that the expected time decreased by 0.97 seconds between each assessment. For testing whether the expected time increased in response to negative achievement-related feedback (that is, needing more time than expected), we again analyzed data from four time points (starting with the immediate expectation before the first receipt of negative feedback). The significant slope indicates that the expected time increased by 5.39 seconds between successive times of measurement. We could only analyze expectation change after the receipt of positive feedback between two times of measurement (comparing expectations before the first better-than-expected trial and the second better-thanexpected trial). The expected time for solving the task decreased by 5.50 seconds, which was a significant change (Table 1 ). We found that $45.31 \%$ of the withinindividual variation of expectations in the no-feedback condition, $82.63 \%$ in the negative feedback condition, and $82.12 \%$ in the positive feedback condition was associated with linear rate of change in expectations.

We also compared expectation change between the first two iterations with negative feedback and both with positive feedback. The amount of change did not differ $(M$ $=5.52,95 \%$ confidence interval $[95 \% \mathrm{CI}], 4.66$ to 6.37 , vs. $M=-5.50,95 \% \mathrm{CI},-6.85$ to -4.15$)$, while the direction of change differed. 
In Model 2, sex (coded male $=1$, female $=2)$ and grade level (ranging from 3 to 6) were added as level 2 predictors. We used grade level rather than age as an independent variable because grade level was distributed equally in our sample. The primary effects of interest were the cross-level interactions between time of measurement and sex or grade level. We found an effect of sex on the intercept in the no-feedback condition: Female students expected to need more time for completing the task than male students in the no-feedback condition $(d=0.26)$. However, there were no other significant effects of sex and grade level on the intercept and on the slope (expectation change) (Table 2).

With a general linear model (GLM) analysis, we tested whether the reported levels of assimilation, accommodation, and immunization differed by grade level and sex. To get robust results, we combined third graders and fourth graders as well as fifth graders and sixth graders. We found a significant overall main effect of sex and a significant interaction effect of grade level and sex (Table 3). Analyses on assimilation showed a significant main effect of grade level, with the group third and fourth graders reporting higher assimilation than the group fifth and sixth graders $(M=3.39, S D=0.68$ vs. $M=3.17, S D=$ $0.64, d=0.33)$. The main effect of sex on accommodation indicated that girls reported higher accommodation than boys $(M=2.78, S D=0.67$ vs. $M=2.31, S D=0.85 ; d=$ 0.61 ). In addition, there was a significant interaction effect of sex and grade level. Male third and fourth graders' reported scores ( $M=2.05,95 \% \mathrm{CI}, 1.73$ to 2.36$)$ were lower than the reported accommodation of female third and fourth graders ( $M=2.88,95 \% \mathrm{CI}, 2.65$ to 3.12 ) and female fifth and sixth graders $(M=2.65,95 \% \mathrm{CI}$, 2.40 to 2.91). We also found a main effect of sex on immunization. Girls reported higher immunization than boys $(M=1.03, S D=0.77$ vs. $M=0.74, S D=0.67 ; d=$ $0.28)$.

For analyzing associations of immunization, assimilation, and accommodation with levels and change of expectations, we specified a new level 2 MLM, which included main effects of these three coping styles and time of measurement, as well as interaction effects. Separate analyses were run for the no-feedback, negative-, and positive-feedback conditions. The primary effects of interest were the cross-level interactions between time of measurement and coping styles. No main or interaction effects of the coping styles were found in the no-feedback condition (Table 4). In the negative-feedback condition, we found a main effect of immunization on the intercept and a cross-level interaction of immunization on the slope of expectation change. The negative estimate of 3.02 indicates that immunization was, on average, associated with a lower expected time for completing the task.

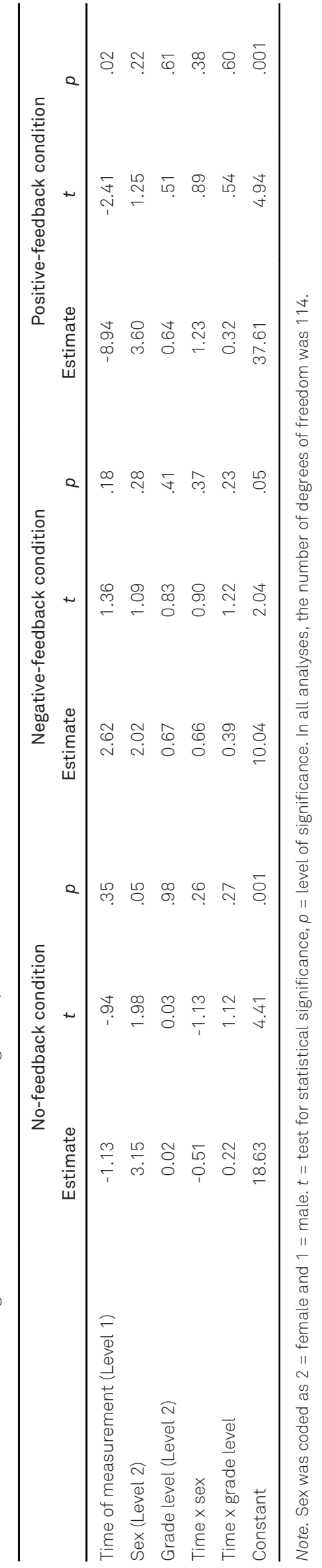




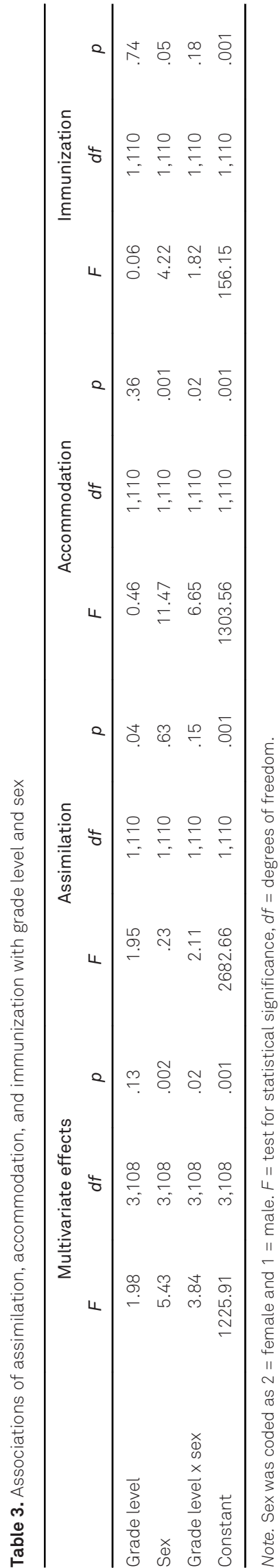

However, the positive interaction term of immunization and time of measurement indicates that the immunization effect became weaker over time. In the last negativefeedback trial, we even found a positive, albeit nonsignificant, association of immunization and increase in the expected time for task completion (estimate $=0.98$ ). The marginally significant interaction effect of time of measurement and accommodation indicates that, over time, accommodation tended to predict a stronger increase in the expected time. Finally, in the positive-feedback condition, we found no main effects of the coping strategies on the intercept. However, there was a significant crosslevel interaction effect of accommodation on the slope (expectation change), indicating that students with higher accommodation scores decreased the expected time less often.

Hypothesis 5 asked whether the associations of grade and sex with expectation change would be mediated by the three ways of coping. Mediation analyses based on Hayes (2013) found no significant mediation effects of accommodation, assimilation, and immunization.

\section{Discussion}

The present study analyzed expectation change and coping with expectation violation in third to sixth graders. Students' expected time to complete the task declined when they did not receive feedback about their achievement, and after performing better than expected. However, we found the reverse after needing more time than expected for completing the task. Expectation change did not vary by grade level or sex. While in the negativefeedback condition, immunization was related to less negative expectations about the time needed for completing the next trial, the protective immunization effect was lost with an increasing number of expectation violations. In addition, accommodation was associated with a less positive and more negative expectation change after receiving better than expected feedback about the previous achievement.

The observed expectation change in the negative- and positive-feedback conditions showed that the manipulation of the achievement feedback had the effects that we had anticipated. The small, but significant decrease in the expected time during the no-feedback trials probably reflects a training effect, as repeated exercise increases achievement, because it is reasonable to expect less time to complete the task with increasing practice (Sohn \& Anderson, 2001). As we observed similar levels of expectation change after the first negative- and positive-feedback trial, the present study did not find an optimistic 
update bias (Garrett \& Sharot, 2017). However, students had completed three trials with lower-than-expected achievement before getting better-than-expected feedback. Thus, the series of previous negative experiences may have limited expectation change after getting betterthan-expected feedback.

Similar to a previous study with first to fourth graders (Pinquart \& Block, 2020), expectation change did not vary by grade level or sex. Age differences may only be found when comparing preschoolers and older children, as observed in two older studies (Parsons \& Ruble, 1977; Stipek \& Hoffman, 1980a). As self-report rating scales on the ViolEx strategies and related constructs did not work with preschoolers (Lessing et al., 2015), we could not include such young children in our study.

The observed main effect of sex on expectations indicates that boys had more optimistic expectations (of needing less time for computing the tasks) in the nofeedback condition. This is in line with results from Parsons and Ruble (1977) as well as Stipek and Hoffman (1980a), although our results indicate that sex differences vanish when students receive clear feedback about their performance.

Until now, not much was known about grade level and sex differences regarding the use of assimilation, immunization, and accommodation in coping with expectation violation. While a recent study observed no grade level or sex differences in these coping strategies (Pinquart \& Block, 2020), we found higher reported levels of assimilation in younger grades. The previous study used another measure of assimilation (based on an analysis of verbalized thoughts) and found that very few of the responses indicated assimilation, which reduced the chance of finding significant group differences. It may be surprising for some readers to find lower levels of reported assimilation among the students from higher grades, because some action resources for fulfilling expectations, such as self-control, tend to increase with age (Tao et al., 2014). However, intrinsic achievement motivation declines, on average, with age, which could translate to a decline in assimilative behavior aimed at fulfilling high achievement expectations (Scherrer \& Preckel, 2019). In the current study, per design, the students' behavior did not affect the chance of expectation confirmation in the feedback conditions. Students from higher grades may have been more aware of this, which could have also reduced their reports of assimilative attempts.

The reported lower accommodation scores of boys, particularly young boys, as compared with girls were in line with results from Parsons and Ruble (1977) as well as Stipek and Hoffman (1980a) on sex differences in expectation change after achievement expectations were dis- 
confirmed. Nonetheless, the present study did not find parallel sex differences in factual expectation change. As girls also reported higher immunization than boys, and as immunization should promote persistence of expectations (Gollwitzer et al., 2018; Rief \& Glombiewski, 2016), effects of sex differences in accommodation and immunization may have cancelled each other out, thus leading to no sex differences in factual expectation change.

A main topic of the present study was whether reported levels of immunization, assimilation, and accommodation would predict expectation change. In line with the ViolEx model (Gollwitzer et al., 2018; Rief \& Glombiewski, 2016), higher levels of immunization were associated with higher achievement expectations in the negativefeedback condition. However, this effect was lost over the successive disconfirmations, indicating that it was easier to immunize one's expectations against a single disconfirming event than against a series of expectation disconfirmations (see also Filipowicz et al., 2018). The data from the last trial with negative feedback even indicated that, in the long run, higher initial immunization may be related to stronger expectation change, which probably demonstrates that the sum of the otherwise gradual expectation change is condensed after realizing that one has to take the expectation violations seriously.

In line with the ViolEx model, reported accommodation was related to (marginally significant) declines in achievement expectations in the negative-feedback condition. At first glance, results on accommodation in the positivefeedback condition may be surprising, as accommodation predicted less expectation updating. However, we had operationalized accommodation as willingness to reduce one's achievement expectations rather than willingness to change these expectations in both directions. Thus, it is not surprising that higher accommodation scores predicted lower improvements of achievement expectations in the positive-feedback condition (i.e., less decline in the expected time to complete the task). Finally, the lack of statistical effects of assimilation on expectation change may be explained by the fact that, per study design, interindividual differences in assimilative behavior did not have an effect on expectation confirmation in the next trials.

\section{Limitations and Conclusions}

Although the present study overcame many limitations of the previous study by Pinquart and Block (2020) by adding better-than-expected events to the worse-thanexpected events, by assessing expectation change over a larger number of trials, and by using multi-item measures for assessing the ViolEx strategies, some limitations of the present study should be mentioned. First, as we included only third to sixth graders, the age and/or grade level range may have been too narrow for finding age and/or grade level differences. However, tasks and measures must be adapted to the verbal and cognitive skills of the children, which limits the age range for applying identical measures. Second, we could not rely on a previously validated measure of the ViolEx strategies among children. Third, as we needed the no-feedback condition as a baseline for building expectations, and as we did not want students to end their participation immediately after experiencing a number of worse-than-expected events, all participants completed the three feedback conditions in the same order. Reactions toward unexpected negative or positive events may differ depending on the kind of events experienced beforehand. Fourth, our results were based on a specific task and should be replicated with other experimental tasks. Finally, the participants did not have any opportunities to influence future achievement feedback. Effects of assimilation on persistence and change of expectations may become obvious if the study design would allow for such opportunities.

Despite these limitations, several conclusions can be drawn. First, we conclude that expectations of third to sixth graders are similarly responsive to better-thanexpected as well as worse-than-expected achievement feedback. Second, the present study adds to the growing evidence that the use of immunization and accommodation after expectation violation does not systematically vary across the first school years. However, we conclude from our study that after worse-than-expected achievement, older children are less likely to use assimilation, at least as long as they do not experience an effect of assimilation on their next outcomes. Future studies should test whether this age difference persists when students experience effects of assimilation on expectation confirmation. When using larger samples, three-way interaction effects of age (grade level), coping, and time of measurement should also be tested. Third, similar to the study by Pinquart and Block (2020), we found that elementary school children protect their positive achievement expectations by using immunization against discrepant feedback. However, we conclude from the present study that the effect of immunization vanishes after being confronted with repeated expectation disconfirmation and that there may even be a condensed adjustment of expectations after realizing that worse-than-expected achievements were not single exceptions from the rule. Fourth, with regard to future research, we need more valid assessments of the ViolEx strategies among children and adolescents. For example, separate measures could be developed on coping with worse-than-expected and better-than-expected events. Similarly, researchers may 
develop parental reports and (adolescent) self-reports on dispositional use of immunization, assimilation, and accommodation, similar to the parental reports on children's tenacious goal pursuit and flexible goal adjustment. In addition, researchers may provide situational cues that promote the use of an individual way of coping (e.g., Kube et al., $2018</$ litr $</$ ) and check whether this affects children's self-reported coping and their expectation change. For predicting the use of assimilation and accommodation, future studies should also assess attributions, as, for example, perceptions of internal and changeable sources of unexpected negative feedback may promote the use of assimilative behavior. Finally, the observed changing effects of immunization indicate that future research should also assess how characteristics of expectations (e.g., perceived importance, amount of previous confirmations), disconfirming events (e.g., size of the discrepancy and number of discrepant events), and the broader situation (e.g., whether expectations are shared with peers) affect coping with expectation violation.

\section{Electronic Supplementary Material}

The electronic supplementary material is available with the online version of the article at https://doi.org/10. $1026 / 0049-8637 / \mathrm{a} 000222$

ESM 1. Examples of the experimental task

ESM 2. Assessment of coping with expectation violation

ESM 3. Factor loadings of the coping items

ESM 4. Intercorrelations of main study variables

ESM 5. Means and standard deviations of the main study variables

\section{References}

Filipowicz, A., Valadao, D., Anderson, B., \& Danckert, J. (2018). Rejecting outliers: Surprising changes do not always improve belief updating. Decision, 5(3), 165-176. https://doi.org/10. 1037/dec0000073

Garrett, N., \& Sharot, T. (2017). Optimistic update bias holds firm: Three tests of robustness following Shah et al. Consciousness and Cognition, 50(1), 17-22. https://doi.org/10.1016/j.concog. 2016.10.013

Gollwitzer, M., Thorwart, A., \& Meissner, K. (2018). Editorial: Psychological responses to violations of expectations. Frontiers in Psychology, 8, 2357. https://doi.org/10.3389/fpsyg.2017. 02357

Hacker, D. J., Bol, M., Hogan, D., \& Rakow, E. A. (2000). Test prediction and performance in a classroom context. Journal of Educational Psychology, 92(1), 160-170. https://doi.org/10. 1037/0022-0663.92.1.160
Hayes, A. F. (2013). Introduction to mediation, moderation, and conditional process analysis: A regression-based approach. Guilford Press.

Kruger, J. M., \& Dunning, D. (1999). Unskilled and unaware of it: How difficulties in recognizing one's own incompetence lead to inflated self-assessments. Journal of Personality and Social Psychology, 77(6), 1121-1134. https://doi.org/10.1037/00223514.77.6.1121

Kube, T., Rief, W., Gollwitzer, M., Gärtner, T., \& Glombiewski, J. A. (2018). Why dysfunctional expectations in depression persist: Results from two experimental studies investigating cognitive immunization. Psychological Medicine, 22(9), 1532-1544. https://doi.org/10.1017/S0033291718002106

Kube, T., Rief, W., Gollwitzer, M., \& Glombiewski, J. A. (2018). Introducing an Experimental Paradigm to investigate Expectation Change (EXPEC). Journal of Behavioral Therapy and Experimental Therapy, 59, 92-99. https://doi.org/10.1016/j. jbtep.2017.12.002

Lessing, N., Mähler, C., \& Greve, W. (2015). Zur Erfassung und Validierung akkommodativer Bewältigungsfähigkeiten im Grundschulalter. Ein Erhebungsinstrument [Measurement and validation of accommodative coping in primary school: A survey instrument]. Zeitschrift für Entwicklungspsychologie und Pädagogische Psychologie, 47(4), 210 - 218. https://doi.org/10.1026/ 0049-8637/a000138

Moser, J. S., \& Schroder, H. S. (2012). Making sense of it all? Cognitive and behavioral mechanisms needing clarification in the meaning maintenance model. Psychological Inquiry, 23(4), 367 - 373. https://doi.org/10.1080/1047840X.2012.721338

Muenks, K., Wigfield, A., \& Eccles, J. S. (2018). I can do this! The development and calibration of children's expectations for success and competence beliefs. Developmental Review, 48(1), 24-39. https://doi.org/10.1016/j.dr.2018.04.001

Parsons, J. E., \& Ruble, D. N. (1977). The development of achievement-related expectancies. Child Development, 48(3), $1075-$ 1079. https://doi.org/10.2307/1128364

Pinquart, M., \& Block, H. (2020). Coping with broken achievementrelated expectations in students from elementary school: An experimental study. International Journal of Developmental Science, 14(1-2), 9-17. https://doi.org/10.3233/DEV-200001

Pinquart, M., \& Ebeling, M. (2020). Students' expected and actual academic achievement: A meta-analysis. International Journal of Educational Research, 100, 101524. https://doi.org/10.1016/ j.ijer.2019.101524

Rief, W., \& Glombiewski, J. A. (2016). Expectation-focused psychological interventions. Verhaltenstherapie, 26(1), 47-54. https:// doi.org/10.1159/000442374

Roese, N. J., \& Sherman, J. W. (2007). Expectancy. In E. T. Higgins \& A. W. Kruglanski (Eds.), Social psychology: Handbook of basic principles (2nd ed., pp. $91-115)$. Guilford Press.

Scanlan, T. K., \& Passer, M.W. (1981). Determinants of competitive performance expectancies of young male athletes. Journal of Personality, 49(1), 60-74. https://doi.org/10.1111/j.14676494.1981.tb00846.x

Scherrer, V., \& Preckel, F. (2019). Development of motivational variables and self-esteem during the school career: A metaanalysis of longitudinal studies. Review of Educational Research, 89(2), 211-258. https://doi.org/10.3102/ 0034654318819127

Singer, J. D. (1998). Using SAS PROC MIXED to fit multilevel models, hierarchical models, and individual growth models. Journal of Educational and Behavioral Statistics, 24(4), $323-$ 355. https://doi.org/10.3102/10769986023004323

Singer, J. D., \& Willett, J. B. (2003). Applied longitudinal data analysis: Modeling change and event occurrence. Oxford University Press. 
Sohn, M. H., \& Anderson, J. R. (2001). Task preparation and task repetition: Two-component model of task switching. Journal of Experimental Psychology: General, 130(4), 764-778. https:// doi.org/10.1037/0096-3445.130.4.764

Stipek, D. J., \& Hoffman, J. M. (1980a). Development of children's performance-related judgments. Child Development, 51(3), 912 - 914. https://doi.org/10.2307/1129485

Stipek, D. J., \& Hoffman, J. M. (1980b). Children's achievementrelated expectancies as a function of academic performance histories and sex. Journal of Educational Psychology, 72(6), 861 - 865. https://doi.org/10.1037/0022-0663.72.6.861

Tao, T., Wang, L., Fan, C., \& Gao, W. (2014). Development of selfcontrol in children aged 3 to 9 years: Perspective from a dualsystems model. Scientific Reports, 4, 7272. https://doi.org/10. 1038/srep07272

\section{Acknowledgement}

This study was conducted by the research training group Expectation Maintenance Versus Change in the Context of Expectation Violations: Connecting Different Approaches and funded by the German Research Foundation (Deutsche Forschungsgemeinschaft, DFG Project number 290878970-GRK 2271).

\section{Funding}

Open access publication enabled by Philipps-University of Marburg.

\section{ORCID}

Martin Pinquart

(iD)https://orcid.org/0000-0003-4496-4250

\section{Prof. Dr. Martin Pinquart}

Department of Psychology Philipps-University of Marburg

Gutenbergstrasse 18

35032 Marburg

pinquart@staff.uni-marburg.de 